\title{
Persistence of simple and contingent motion aftereffects
}

\author{
OLGA EIZNER FAVREAU \\ University of Montreal, Montreal, Quebec, Canada
}

\begin{abstract}
Jones and Holding (1975) showed that orientation-contingent color aftereffects can persist for at least 3 months, but are depleted by repeated testing. We applied the same paradigm to a simple motion aftereffect (MAE) and found that it can persist for up to 1 week and is only slightly diminished by testing. It was further found that simple MAEs appear to persist longer than color-contingent MAEs, although when procedures for inducing and measuring both kinds of aftereffect are equalized, contingent MAEs last longer. Finally, no tendency was found for color-contingent MAEs to diminish with repeated testing. Although both simple and color-contingent MAEs can be relatively persistent, there are certain differences between them. Furthermore, contingent aftereffects should not be considered interchangeable, as there appear to be large differences in the persistence of orientation-contingent color aftereffect and color-contingent MAEs.
\end{abstract}

The first report of a contingent aftereffect was made by McCollough in 1965 . She exposed observers to alternating presentations of vertical and horizontal square-wave gratings in orange and blue light, respectively. After 4 min of cumulated exposure, observers reported that achromatic vertical gratings appeared slightly bluish and achromatic horizontal gratings seemed tinged with orange. This is an orientation-contingent color aftereffect and is now known by the discoverer's name. Other contingent aftereffects have since been discovered. For example, by using appropriate adapting conditions, a color aftereffect can be made contingent on the direction of motion of a grating (Hepler, 1968; Stromeyer \& Mansfield, 1970) or on its spatial frequency (Lovegrove \& Over, 1972). Conversely, apparent orientation (Held \& Shattuck, 1971) or direction of motion can be made contingent on color (Favreau, Emerson \& Corballis, 1972; Mayhew \& Anstis, 1972).

It has been observed that contingent aftereffects can be quite persistent. McCollough (1965) noted that the orientation-contingent color aftereffect is still available at least 1 hour after the initial adaptation period, well beyond the usual persistence of complementary color afterimages. Hepler (1968) reported that motion-contingent color aftereffects are still seen about $24 \mathrm{~h}$ after adaptation. Favreau et al. (1972) found that color-contingent motion after-

This research was supported by Grant A 9846 from the Natural Sciences and Engineering Research Council of Canada. I would like to thank Lise Guilbault and Jacques Labelle for assistance with Experiment 1, and Raymonde Guindon, Yves Lespérance, and Christiane Provençal, who worked on Experiments 2, 3, and 4 as part of a laboratory course project. Reprint requests should be addressed to Olga Eizner Favreau, Département de Psychologie, Université de Montréal, Québec, H3C 3J7, Canada. effects, too, can persist for at least $24 \mathrm{~h}$. Stromeyer and Mansfield (1970) reported a few instances in which motion-contingent color aftereffects were still present 5 to 6 weeks after the initial adaptation period. A number of authors have reported that the McCollough effect can persist for fairly long periods of time (e.g., Mackay \& Mackay, 1975; Riggs, White, \& Eimas, 1974; Shute, 1977), but by far the longest persistence period has been reported by Jones and Holding (1975). These authors, in a systematic exploration of the persistence of the McCollough effect, reported that it is still available at half its initial strength at least $2040 \mathrm{~h}$ (or 3 months) after $15 \mathrm{~min}$ of exposure to the adapting stimulus.

When McCollough first discovered the orientationcontingent color aftereffect, she explained it in terms of neural fatigue in double-duty cortical feature detectors similar to those which had been described by Hubel and Wiesel (1959). Sutherland (1961) had earlier attributed simple aftereffects to the same process. As it became evident that contingent aftereffects could persist beyond the known limits of neural fatigue, it appeared necessary to view them as qualitatively different from simple aftereffects. It has been suggested (e.g., Leppmann, 1973; Murch, 1976; Skowbo, Gentry, Timney, \& Morant, 1974) that contingent aftereffects are formed because the two components of the contingency (e.g., color and orientation or color and motion) become classically conditioned to each other. One problem which arises from an analogy with classical conditioning is that, in contingent aftereffects, either member of the contingency can evoke the perception of the negative of the other. Thus, for example, Favreau et al. (1972) found that after exposure to red and green spirals alternately rotating in opposite directions observers saw color- 
contingent motion aftereffects when they looked at stationary colored spirals and motion-contingent color aftereffects when they looked at achromatic rotating spirals. This interchangeability of $\mathrm{CS}$ and UCS is not characteristic of classical conditioning.

Harris and Frome (Note 1) have pointed out that a classical conditioning explanation of the persistence of contingent aftereffects depends for its validity on the presence of two components in a contingency which can become conditioned to each other. Simple aftereffects, by implication, would not be so persistent because they lack associable components. This view, then, requires that simple aftereffects not persist beyond the relatively brief period necessary for recovery from neural fatigue. There is, however, evidence that some simple aftereffects can actually persist for fairly long periods. In fact, as long ago as 1969, Masland reported that $24 \mathrm{~h}$ after $15 \mathrm{~min}$ of exposure to a rotating spiral, observers report seeing a motion aftereffect. This was confirmed in 1972 by Kalfin and Locke. Simple aftereffects based on spatial frequency lasting up to several hours have been reported by Blakemore, Nachmias, and Sutton (1970) and by Heggelund and Hohmann (1976). Harris and Frome (Note 1) reported that a size illusion aftereffect can persist for about 1 day after a suitably long adaptation period. Simple aftereffects can generally be induced after very short periods of exposure-only a few seconds suffice to induce a motion aftereffect. By contrast, in order to induce contingent aftereffects it is necessary to employ relatively long adaptation periods. It would seem, then, that equally lengthy adaptation periods may also induce very persistent simple aftereffects as was seen in the work of Masland (1969) and of Harris and Frome (Note 1). Thus, the difference in persistence between contingent and simple aftereffects that has been alleged to exist may be due to the different lengths of the adaptation periods rather than to qualitatively different underlying processes.

The purpose of the following experiments is to explore some aspects of simple and contingent aftereffects. Duration and persistence are used to determine whether these parameters can permit one to differentiate between these two kinds of aftereffect, especially with a view towards evaluating the relative merits of the fatigue and association theories. (Duration denotes the length of time on any one test that the MAE is perceptible; persistence refers to how long after adaptation the MAE can be observed.) The work of Jones and Holding (1975) on the McCollough effect showed that not only can it persist for very long periods, but also that the persistence depends on the number of times an observer views the test stimulus. The effect is stored much longer if observers are given only one test at any given interval than if they have a series of tests.
Each test appears to deplete the strength of the aftereffect. With tests at $0,8,24,56$, and $120 \mathrm{~h}$ following adaptation, the effect declined steadily, whereas when observers were tested at only one of these intervals, there was no appreciable decrement, even at $120 \mathrm{~h}$. The authors interpret these findings as further evidence for an association model, for tests can be conceived of as extinction trials, when the presentation of a test is the equivalent of presenting a CS without the UCS.

In Experiment 1, the Jones and Holding paradigm is applied to the motion aftereffect (MAE) to explore the limits of persistence of that aftereffect when the adaptation period is $15 \mathrm{~min}$ - the same as that used by Jones and Holding. The intent is to see whether these simple noncontingent motion aftereffects can be as persistent as the McCollough effect and whether they, too, are depleted by repeated tests.

\section{EXPERIMENT 1}

\section{Method}

Observers. The observers were 96 men and women who were recruited from among students, faculty, and staff at the University of Montreal. All had normal or corrected vision.

Apparatus and Procedure. Observers were seated $1.6 \mathrm{~m}$ from a 20-cm 33/4-turn black-and-white spiral which was driven by a variable-speed motor. The spiral subtended $2.1^{\circ}$ of visual angle. A switch connected to a timer permitted an observer to indicate the duration of motion aftereffects on each test trial.

During the adaptation period, all observers watched the spiral rotate at 26 revolutions/min for $15 \mathrm{~min}$. Half of the observers saw clockwise rotation (expansion), and the other half saw counterclockwise rotation (contraction). They were all instructed to fixate the spiral's center during both adaptation and testing. They were divided into eight equal groups. All 12 observers in the repeated tests group were tested $0,4,7,24,48,96$, and $168 \mathrm{~h}$ after adaptation. The remaining observers were in the single test group and were divided into seven subgroups, each of which was tested at only one of these seven intervals.

For each test, observers were asked to look at the center of the stationary spiral and to indicate the duration of the aftereffect by pressing the timer switch and keeping it depressed until the aftereffect was no longer apparent. They were also asked to indicate the direction of the aftereffect (apparent expansion or contraction). At each test interval, there were two identical tests (referred to, respectively, as Test 1 and Test 2 ). In the 0-delay condition, the first of these tests followed immediately after adaptation and the second was 7 min later. At all other delays, there were also two tests separated by a 7 -min interval. One reason for testing twice at each interval is that earlier work had indicated that, with lengthy adaptation periods, the MAE is composed of two components-one which decays rapidly (within about $5 \mathrm{~min}$ ) and another of much longer persistence and which decays more slowly (Masland, 1969). This procedure also permits us to estimate whether successive tests or the passage of time contribute more to the decay of the aftereffect, for one can compare the differences between successive tests when they are separated by only a few minutes with the differences between tests which are separated by hours or days.

\section{Results}

Table 1 shows the medians for each group at each test interval. All observers reported aftereffects in the 
Table 1

Median MAE Durations in Seconds and Standard Errors at Each Test Interval for Single- and Repeated-Test Groups in Experiment 1

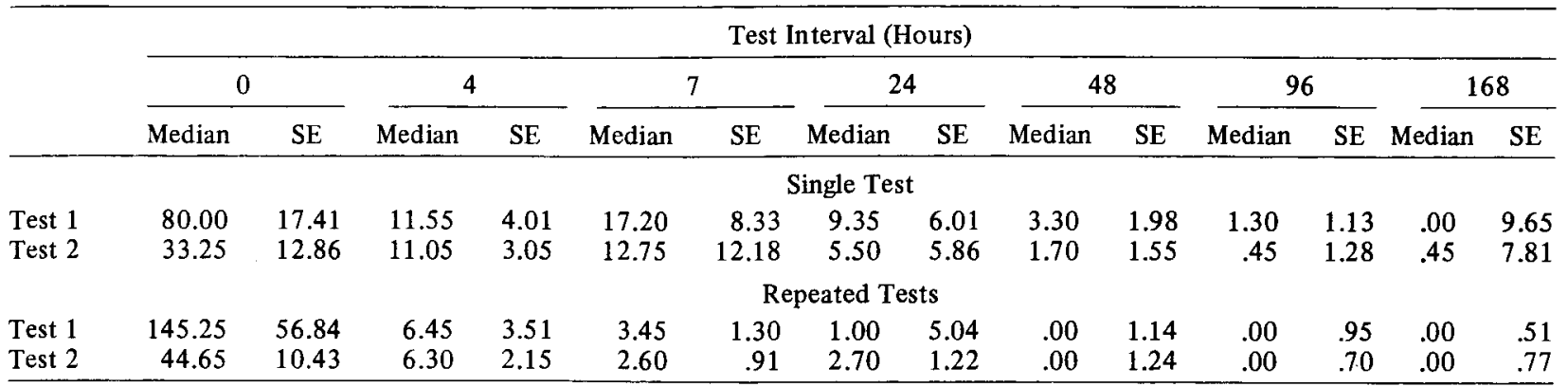

appropriate direction. The difference between the single and repeated test groups at each interval was evaluated using the Wilcoxon rank sum test for independent samples (Ferguson, 1971) ${ }^{1}$ These differences are statistically significant only at $7 \mathrm{~h}$ (Test 1 , $\mathrm{p}<.005$; Test $2, \mathrm{p}<.05$ ) and at $48 \mathrm{~h}$ (Test 1 only, $\mathrm{p}<.025$ ). For all other test intervals, although the differences are in the expected direction, with longer motion aftereffects for the single test group, the differences between the groups fail to reach the conventional levels of statistical significance. One possible problem is that, as can be seen in Table 1 , the baseline measure on Test 1 at 0 delay was $145.25 \mathrm{sec}$ for the repeated tests group but only $80.00 \mathrm{sec}$ for the single test groups. This difference, however, was not significant. Furthermore, by Test $2,7 \mathrm{~min}$ later, the difference was much reduced (44.65 and $33.25 \mathrm{sec}$, respectively). It thus seems unlikely that the initial insignificant difference between the groups could have seriously distorted the subsequent comparisons between treatments.

In the single test group, 6 of 12 observers reported seeing motion aftereffects $168 \mathrm{~h}$ (1 week) after adaptation. In order to further test the limits of persistence of this effect, one more group of 10 observers was adapted and tested $336 \mathrm{~h}$ ( 2 weeks) later; none of these reported seeing motion aftereffects. Thus, we can conclude that, with 15 min of adaptation exposure with the stimulus parameters used in this experiment, the simple motion aftereffect can persist for at least 1 week but not for as long as 2 weeks.

In this experiment, each observer was tested twice at each test session. This makes it possible to evaluate the extent to which decreases in MAE can be attributed to testing or to the passage of time by comparing the differences in MAE duration between Tests 1 and 2 on the same test session with the differences between Test 2 of one session and Test 1 of the following session. If the passage of time contributes more to MAE decreases than does testing, then we should observe larger decreases between Test 2 of one session and Test 1 of the following session than between Tests 1 and 2 on the same session. The results show that the mean of the differences between Tests 1 and 2 at each interval for the single test group is $8.22 \mathrm{sec}$, whereas the mean of the differences between each Test 2 and the subsequent Test 1 is $3.67 \mathrm{sec}$. The corresponding values for the repeated tests group are 14.27 and $7.56 \mathrm{sec}$. In neither case is this difference statistically significant. However, the fact that there is a tendency for the Test 1 -Test 2 difference to be greater than the Test 2-Test 1 difference implies that the decline in the duration of the MAE occurs both as a function of the number of tests as well as of the passage of time.

\section{EXPERIMENT 2}

The results of the above experiment tell us a number of things about the simple MAE. We can see that the effect is quite persistent (at least 1 week) but not nearly as persistent as the 3-month-long McCollough effect that was measured by Jones and Holding (1975). Also, although the motion aftereffect does appear to be somewhat diminished by testing as well as by the passage of time, we did not find the very clear distinction obtained by Jones and Holding.

One question raised by the above results is whether the relatively shorter persistence of the motion aftereffect compared with the McCollough effect is due to the fact that it is a simple aftereffect rather than a contingent one. That is, would establishing a contingency increase the persistence? Experiment 2 was performed in order to provide an answer to this question.

\section{Method}

Observers. The 80 observers who participated in this experiment were recruited from among students, faculty, and staff at the University of Montreal. All had normal or corrected vision.

Apparatus and Procedure. A single-throw 33/4-turn arithmetic spiral was cut out of black matte paper and pasted onto an $8-\mathrm{cm}$ diam white cardboard disk which had been covered with an irregular stippled black-and-white pattern (Letratone, No. LT134). 
The spiral and stippled background each covered about $50 \%$ of the area of the disk.

The disk was mounted vertically on a motor-driven shaft. The motor was hidden behind a white screen. A hole cut in the screen permitted the shaft to project through it, so that the observers saw only the spiral against the screen. The spiral subtended $3^{\circ}$ of visual angle and was rotated at 5 revolutions/min during the adaptation period.

Slides made from Kodak Wratten filters 26 (red), 61 (green), and 96 (neutral density, .8) were used to project red, green, and white light, respectively, onto the spiral and screen. The light source was a tungsten light bulb in a Kodak Ektagraphic projector. The resultant mean luminances on the entire spiral, as measured by a Spectra Spot Meter, were $21 \mathrm{~cd} / \mathrm{m}^{2}$ for the red light, $28 \mathrm{~cd} / \mathrm{m}^{2}$ for the green light, and $25 \mathrm{~cd} / \mathrm{m}^{2}$ for the neutral light. A lever connected to a Digital Stop Clock permitted the observers to indicate the duration of the aftereffect.

The observers were seated at a distance of $1.5 \mathrm{~m}$ from the spiral. Before the exposure to the spiral, they were adapted for $5 \mathrm{~min}$ to the prevailing illumination in the room, which came from light projected through the neutral density filter. During this preadaptation period, the spiral was hidden from view.

There were two main groups of observers: a color-contingent motion aftereffect (CCMAE) group of 40 , and a simple motion aftereffect (SMAE) group of 40 . During the motion adaptation period, the observers in the CCMAE group looked at the rotating spiral illuminated in red light for $60 \mathrm{sec}$. Then, simultaneously, the direction of rotation was reversed and the color of the light was changed to green. This cycle was repeated eight times to give a total of $16 \mathrm{~min}$ of adaptation time. Order of presentation of color and direction of rotation were counterbalanced within groups.

Observers in the SMAE group looked at the spiral rotating in one direction only while it was illuminated by light projected through the neutral density filter for a full $16-$ min period. Half saw counterclockwise rotation.

After the motion adaptation period, each of the two groups was subdivided into five groups who were tested, respectively, at a postadaptation interval of $7 \mathrm{~min}, 24 \mathrm{~h}, 48 \mathrm{~h}, 96 \mathrm{~h}$, or $168 \mathrm{~h}$. Each observer was tested at only one of these intervals. The shortest interval was set at $7 \mathrm{~min}$ because of previous evidence that color-contingent motion aftereffects do not reach full strength until approximately this delay period (Favreau, 1976).

For the SMAE group, testing proceeded as follows: the observers were instructed to fixate the center of the stationary spiral and to depress the lever which activated the timer for as long as they saw any apparent motion. They also indicated verbally the apparent direction of rotation in the stationary spiral. This was a forced choice guess-if they claimed to see no motion, they were nevertheless asked to guess in what direction the spiral appeared to be turning.

The testing for the CCMAE group was similar, except that each observer was tested twice-once with the stationary spiral in one color light (Test 1 ) and another test immediately after that with the other color (Test 2).

\section{Results}

On each test, each observer could make two responses: (1) a statement of the direction in which the stationary spiral appeared to rotate, and (2) an indication of the duration, if any, of the apparent motion. The two responses were combined to produce a single score as follows: When the reported direction was appropriate for a negative aftereffect (i.e., opposite to the direction of rotation), the duration was recorded as a positive value. Conversely, when the reported direction was inappropriate, the duration was signed negatively. Thus, for example, an observer who reported an appropriate MAE with a duration of $10 \mathrm{sec}$ was assigned a score of +10.0 . Observers who did not record a duration were assigned scores of +0 or -0 , depending on the appropriateness of the forced choice directional response. The forced-choice procedure permitted us to include the zero scores in computing the results, as, by assigning positive or negative values to zero scores, we can accord them the lowest ordinal ranks and then include them in a nonparametric statistical test (described below) to determine whether the response differed significantly from chance.

At each test interval, the observers in the CCMAE condition made two judgments, one for each color. The scores of these two judgments were added together to give one score for each session.

For each test session, in order to determine whether the distribution of scores differed significantly from chance, each score was compared with a theoretical score of unsigned zero, using the Wilcoxon matched pairs signed-ranks test (Ferguson, 1971). All of the durations were ranked regardless of sign, the lowest ranks being assigned to the zeros. The sum of ranks of the negative durations $(\mathrm{W}-$ ) was calculated and $\mathrm{W}$ - was referred to a table of critical values of $\mathrm{W}$ for the Wilcoxon test (Ferguson, 1971).

The results are summarized in Table 2 . This table displays the means for each test interval of the simple and color-contingent motion aftereffects. In this experiment, significant simple MAEs occur up to, but not beyond, $96 \mathrm{~h}$, whereas significant colorcontingent MAEs only occur up to $48 \mathrm{~h}$ following adaptation. It should be noted, parenthetically, that the longer persistence of the simple MAE in Experiment 1 compared with that in Experiment 2 is probably attributable to the different stimulus parameters in the two experiments (the sizes and speeds of rotation of the spirals differed). It would appear that the obvious conclusion to draw regarding the difference between simple and color-contingent

Table 2

Median Durations in Seconds and Standard Errors of Simple and Color-Contingent MAEs at Each Test Interval

\begin{tabular}{|c|c|c|c|c|c|c|c|c|c|c|c|c|c|c|c|}
\hline \multirow[b]{3}{*}{ Group } & \multicolumn{15}{|c|}{ Test Interval } \\
\hline & \multicolumn{3}{|c|}{$7 \mathrm{~min}$} & \multicolumn{3}{|c|}{$24 \mathrm{~h}$} & \multicolumn{3}{|c|}{$48 \mathrm{~h}$} & \multicolumn{3}{|c|}{$96 \mathrm{~h}$} & \multicolumn{3}{|c|}{$168 \mathrm{~h}$} \\
\hline & Median & $\mathrm{p}^{*}$ & $\mathrm{SE}$ & Median & $\mathrm{p}^{*}$ & SE & Median & p* & SE & Median & $\mathrm{p}^{*}$ & $\mathrm{SE}$ & Median & $\mathrm{p}^{*}$ & SE \\
\hline SMAE & 5.41 & .005 & 4.41 & 1.62 & .025 & .75 & +0 & .005 & .82 & 1.10 & .025 & .92 & -0 & n.s. & 2.31 \\
\hline CCMAE & 5.42 & .005 & 4.00 & 2.09 & .05 & .70 & .88 & .025 & .56 & -.10 & n.s. & 2.60 & -.00 & n.s. & 1.16 \\
\hline
\end{tabular}

${ }^{*} p<$ value given; $n . s .=$ nonsignificant . 
MAEs is that the former actually persist longer than the latter. However, there are some procedural differences both in induction and testing which make the results not directly comparable.

Consider, for example, the adaptation time. In all cases, observers were adapted for a total of $16 \mathrm{~min}$. However, a question arises as to what is being adapted. According to some contemporary theoretical views, we are adapting neural units that are sensitive to the stimulus dimensions of the display. Presumably, for the SMAE group, this includes units which are sensitive to motion and to edges defined by a luminance difference (in the present context, it is irrelevant whether this sensitivity is to both dimensions combined in the same units or whether it exists in separate units which can become associated with each other). All of these units are adapted for a total of $16 \mathrm{~min}$. Consider, on the other hand, the CCMAE group. In this condition, one set of units sensitive to motion in one direction and to red are being adapted for $8 \mathrm{~min}$ and another set sensitive to motion in the opposite direction and to green are adapted for another $8 \mathrm{~min}$. Thus, for the SMAE, the units which mediate the effect receive a full 16 min of exposure, whereas for the CCMAE there are two sets of units which are each adapted for only $8 \mathrm{~min}$. It is therefore possible that the SMAE was found to be longer because the units involved were adapted for twice as long as the units involved in the CCMAE. Experiment 3 was performed as a test of this possibility.

\section{EXPERIMENT 3}

\section{Method}

There were 24 observers in this experiment, selected in the same way as in the previous experiments. The apparatus was identical to that used in Experiment 2.

The observers were all adapted to a spiral rotating in one direction only and illuminated in red or in green light. Colored rather than white light was used in order to make the conditions as nearly comparable to the contingent MAE as possible. Half of the observers saw one color and the other half saw the other color. Motion was seen during alternate 60 -sec periods. That is, after the observer had looked at the spiral for $60 \mathrm{sec}$, it was hidden from view for a further $60 \mathrm{sec}$. This cycle was repeated eight times. In this way, exposure to the rotating spiral was analogous to the exposure to one of the motion-color pairs which had been experienced by observers in the CCMAE condition of Experiment 2.

The testing procedure was identical to that used for the SMAE group in Experiment 2, with the additional stipulation that obser- vers were tested with the same color light to which they had been adapted. For purposes of testing, the observers were divided into three groups, who were tested, respectively, at intervals of $7 \mathrm{~min}, 24 \mathrm{~h}$, and $48 \mathrm{~h}$. As will be seen in the Results section, no significance MAEs were obtained at the last two intervals, so no additional groups were tested at longer intervals.

\section{Results}

Significant MAEs were obtained only $7 \mathrm{~min}$ after adaptation (the median duration was $8.45 \mathrm{sec}$ ) and had disappeared $24 \mathrm{~h}$ later (the median duration was +0 sec, which did not differ significantly from chance). At $48 \mathrm{~h}$, none of the eight observers recorded any duration time, and of the forced-choice guesses, three were appropriate and five were inappropriate. This contrasts with the color-contingent MAE which we had found to be still available $48 \mathrm{~h}$ following adaptation. Although these results may seem to imply that the color-contingent MAE is indeed more persistent than is the simple MAE when the adaptation conditions are equivalent, there is yet another procedural problem which prevents one from making such an affirmation. It will be recalled that, in order to determine the scores for observers in the CCMAE group, two scores were added together: the scores obtained on each color test (Tests 1 and 2). Thus, while only one sample was taken of the simple MAE, of necessity, two were taken of the color-contingent MAE, and these two were added together. One way to circumvent this difficulty is to examine the scores obtained only on Test 1 or only on Test 2 by the CCMAE group to see whether any single set alone differs significantly from zero. Inspection of Table 3 shows that, in the case of the 7-min test, both Test 1 and Test 2 are significantly different from zero. However, at $24 \mathrm{~h}$, only Test 2 is significant, and at $48 \mathrm{~h}$, neither test alone shows significant MAEs, although both together do. The 96- and 168-h tests are not shown because significant aftereffects were not obtained on these tests. Thus, using these control conditions, there is a tendency for the color-contingent MAE to persist longer than the simple MAE.

\section{EXPERIMENT 4}

Jones and Holding (1975) argued that contingent aftereffects may result from the formation of learninglike associations. One basis for this argument was their finding that repeated tests for the McCollough

Table 3

Medians in Seconds and Standard Errors of Each Color Test at the First Three Delays in the CCMAE Condition in Experiment 2

\begin{tabular}{|c|c|c|c|c|c|c|c|c|c|}
\hline & \multicolumn{9}{|c|}{ Test Delay } \\
\hline & \multicolumn{3}{|c|}{$7 \min$} & \multicolumn{3}{|c|}{$24 \mathrm{~h}$} & \multicolumn{3}{|c|}{$48 \mathrm{~h}$} \\
\hline & Median & $p^{*}$ & SE & Median & $p^{*}$ & SE & Median & $p^{*}$ & SE \\
\hline Test 1 & .85 & .0273 & .96 & +0 & n.s. & .47 & +0 & n.s. & .31 \\
\hline Test 2 & 4.29 & .0039 & 3.54 & .68 & .0195 & .69 & .82 & .0547 & .61 \\
\hline
\end{tabular}

${ }^{*} p=$ value given; $n$. s. = nonsignificant. 
effect led to a more rapid diminution of the strength of the effect than did single tests. If a test is thought of as the presentation of the conditioned stimulus (e.g., achromatic grid or stationary spiral) without the accompanying unconditioned stimulus (color field or motion), then each such test constitutes an extinction trial. Experiment 4 was performed to determine whether repeated tests for the color-contingent MAE have the same effect as do repeated tests for the McCollough effect, as reported by Jones and Holding (1975)-that is, do they diminish the strength of the aftereffect when compared with single tests?

\section{Method}

The method was identical to that used in Experiment 2 for the CCMAE group, except that each observer was tested at every test interval $-7 \mathrm{~min}$, and $24,48,96$, and $168 \mathrm{~h}$. Only four observers served in this experiment, for it became evident, as will be seen below, that the decrease in the magnitude of the effect was no greater than the decrease observed in Experiment 2 with single tests.

\section{Results}

The median color-contingent MAE durations at each test interval were $13.90,9.01,7.27,3.47$, and $0 \mathrm{sec}$ respectively. The corresponding median durations for the single test group in Experiment 2 were $5.42,2.09, .88,-.10$, and $-0 \mathrm{sec}$, respectively. Thus, at each interval, the medians for the multiple test group were actually higher, although this difference was not statistically significant. These results made it obvious that adding more observers to make up a full group of eight observers, even if they had all had inappropriate scores, it would still not have been possible to conclude that the effect is less strong at $96 \mathrm{~h}$ for the repeated tests when compared with the single test group. It therefore seems safe to conclude that multiple testing does not diminish the strength of the color-contingent MAE when compared with single tests.

\section{DISCUSSION AND SUMMARY}

It has been postulated that contingent aftereffects are qualitatively different from simple aftereffects because the former have very long persistence periods. However, the results of Experiment 1 showed that the simple MAE can persist for up to 1 week, thereby extending and confirming the results of earlier reports of relatively long-lasting simple aftereffects. It had, furthermore, been suggested that the long persistence of contingent aftereffects makes it plausible to postulate a learning-like associational process, since traditional fatigue theories of aftereffects could not account for such long persistence. The long persistence of simple aftereffects makes it necessary to reexamine this view. One possibility is to extend the association hypothesis to include simple aftereffects. However, for the time being, a simpler alternative is available. Creutzfeld and Heggelund (1975) exposed adult cats to vertical lines for 1 week. Subsequent electrophysiological measures showed diminished neural response to vertical lines for at least $24 \mathrm{~h}$ after termination of exposure. The results of this research, although they do not tell us just how long the effect persisted, provide an interesting analog for the long-term persistence of negative aftereffects, as they seem to indicate the operation of an adaptive process simpler than association.

In Experiments 2 and 3, attempts were made to compare the relative persistences of simple and contingent MAEs. In order to do so, one must choose an appropriate simple MAE control procedure. The selection of this procedure depends on one's theoretical definition of what constitutes a contingent aftereffect. Consider, for example, the following model, which involves three sets of motion-detecting units, sensitive, respectively, to (a) red, (b) green, and (c) brightness contrast. Each set contains subsets of units sensitive to motion in opposite directions. Following the procedure for inducing a colorcontingent MAE, the a units are biased in one direction, the $b$ units are biased in the opposite direction, and the $c$ units, to which the colored stimuli appeared achromatic, are mutually cancelled. According to this model, the control procedure in Experiment 2 activated the $c$ units for the same period of time that both the $a$ and $b$ units were activated. Since we measured the size of a contingent MAE as (a) $+(b)$, then, from one point of view, the use of a total adaptation time for the simple MAE that is equal to the total for the contingent MAE was not inappropriate. However, since it is likely that (c) contains more units, it is not surprising that the simple MAE was longer.

Again, following the model, in Experiment 3 the units in (a) or (b), but not both, and in (c) would have been adapted. If this were the case, then the simple MAE should have persisted longer than onehalf of the contingent MAE as it would have depended on (a) or (b) $+(c)$, whereas one-half of the contingent aftereffect would depend on (a) or (b) only. However, here the simple aftereffect violated the prediction of the model, and, in fact, persisted much less long than one of the halves of the contingent aftereffect. This could be taken as evidence that the simple MAE is qualitatively different from the color-contingent MAE, or that we have chosen the wrong model for comparison.

In fact, the difficulties with the two control conditions point out the more fundamental problem of the theoretical definition of just what constitutes a contingent MAE. Normally, to induce a contingent aftereffect, pairs of "opposite" stimuli are presented 
in alternation. However, Stromeyer (1969), presenting only one pairing of color and orientation, induced a relatively strong color aftereffect in the orientation that was paired with color, and a very weak complementary color aftereffect in the orthogonal orientation. This indicates that there may be an enhancing interactive process among the color and configurational dimensions. In another vein, Favreau (1976) has shown that brightness contrast in the adapting stimuli can exert a suppressive effect on the colorcontingent MAE. The existence of these kinds of interactions makes it difficult, if not impossible, to select an appropriate simple aftereffect control condition.

What, then, do the results of this study permit us to conclude regarding the comparison of simple and contingent aftereffects? Using two of the more obvious simple MAE control procedures, we found, in one case, that the simple MAE can be longer, and in the other, that it can be shorter than the color-contingent MAE. One could go on trying out different control procedures until one is found which matches the persistence of the contingent aftereffect, but that is hardly likely to shed any light on the matter. Prima facie, the control used in Experiment 3 is the most appropriate because any other control would require postulating assumptions about the nature of contingent aftereffects in which, by definition, they would be different from simple aftereffects. In this connection, it is interesting to note that Walker (1978), using similar procedures for inducing simple and contingent proprioceptive aftereffects, obtained results analogous to those reported here. In summary, the results of Experiment 3 lend support to the view that the mechanisms underlying contingent aftereffects are qualitatively different from simple aftereffects, although it is not yet possible to specify the nature of this difference.

Whatever the nature of this mechanism, the results of Experiment 4 do not support the view that it is analogous to conditioning, as there was no tendency for the color-contingent MAE to be diminished by repeated tests, thus failing to confirm the hypothesis that testing is a form of extinction. In fact, the results of Experiments 1 and 4 regarding the effects of repeated testing are opposite to what a conditioning theory would predict. That is, the simple MAE is depleted by repeated testing, whereas the contingent MAE is not, so that if there is extinction, it fails to occur in a situation in which associable elements are available, and occurs instead in a situation in which such elements are less readily obvious. The various differences between the present findings and those of Jones and Holding (1975) indicate that it may be erroneous to view different kinds of contingent aftereffects as interchangeable with each other. Rather, the differences may reflect differences between motion-detecting and pattern-detecting systems.

\section{REFERENCE NOTE}

1. Harris, C. S., \& Frome, F. What's so special about contingent aftereffects? Paper presented to the meeting of the Canadian Psychological Association in Toronto, 1976.

\section{REFERENCES}

Blakemore, C., Nachmias, J., \& Sutron, P. The perceived spatial frequency shift: Evidence for frequency-selective neurones in the human brain. Journal of Physiology, 1970, 210, 727-750.

Creutzfeld', O. D., \& Heggelund, P. Neural plasticity in visual cortex of adult cats after exposure to visual patterns. Science, 1975, 188, 1025-1027.

Favreau, O. E. Interference in colour-contingent motion aftereffects.Quarterly Journal of Experimental Psychology, 1976, 28, 553-560.

Favreau, O. E., Emerson, V. F., \& Corballis, M. C. Motion perception: A color-contingent after-effect. Science, 1972, 176, $78-79$.

Ferguson, G. A. Statistical analysis in psychology and education. New York: McGraw-Hill, 1971.

Heggelund, P., \& Hohmann, A. Long-term retention of the "Gilinsky-Effect." Vision Research, 1976, 16, 1015-1017.

Held, R., \& Sh Aттuck, S. Color and edge-sensitive channels in the human visual system: Tuning for orientation. Science, 1971, 174, 314-316.

He PLE R, N. Color: A motion-contingent color aftereffect. Science, 1968, 162, 376-377.

Hubel, D. H., \& Wiesel, T. N. Receptive fields of single nuerones in the cat's striate cortex. Journal of Physiology, $1959,148,574-591$.

Jones, P. D., \& Holding, D. H. Extremely long-term persistence of the McCollough effect. Journal of Experimental Psychology: Human Perception and Performance, 1975, 4, 323327.

Kalfin, K., \& Locke, S. Evaluation of long term visual motion after-image following monocular stimulation. Vision Research, $1972,12,359-361$.

LePpMANN, P. K. Spatial frequency dependent chromatic aftereffects. Nature, 1973, 242, 411-412.

Lovegrove, W. J., \& Over, R. Color adaptation of spatial frequency detectors in the human visual system. Science, 1972, 176, 541-543.

Mackay, D. M., \& Mackay, V. Dichoptic induction of McCollough-type effects. Quarterly Journal of Experimental Psychology, 1975, 27, 225-233.

Masland, R. H. Visual motion perception: Experimental modification. Science, 1969, 165, 819-821.

Mayhew, J. E. W., \& Anstis, S. M. Movement aftereffects contingent on color, intensity, and pattern. Perception \& Psychophysics, 1972, 12, 77-85.

McCollough, C. Color adaptation of edge detectors in the human visual system. Science, 1965, 149, 1115-1116.

Murch, G. M. Classical conditioning of the McCollough effect: Temporal parameters. Vision Research, 1976, 16, 615-619.

Riggs, L. A., White, K. D., \& Eimas, P. D. Establishment and decay of orientation-contingent aftereffects of color. Perception \& Psychophysics, 1974, 16, 535-542.

Shute, C. C. D. Strength and decay of McCollough effects. Journal of Physiology, 1977, 268, 34P-36P.

Skowbo, D., Gentry, T., Timney, B., \& Morant, R. B. The McCollough effect: Influence of several kinds of visual stimulation on decay rate. Perception \& Psychophysics, 1974, 16, $47-49$. 
Stomizer. C. F. Further studies of the McCollough effect. Perception \& Psychophysics, 1969, 6, 105-110.

Stomfyer, C. F., \& Mansfield, R. J. W. Colored aftereffects produced with moving edges. Perception \& Psychophysics, 1970, 7. 108-114.

Sutherland, N. S. Figural aftereffects and apparent size. Quarterly Journal of Experimental Psychology, 1961, 13, 222-228.

WALKER, J. T. Simple and contingent aftereffects in the kinesthetic perception of length. Journal of Experimental Psychology: Human Perception and Performance, 1978, 4, 294-301.

\section{NOTE}

1. Nonparametrical statistical tests were used because of the highly skewed and generally nonnormal distribution of the data.

(Received for publication December 20, 1978; revision accepted June 15, 1979.) 